$\begin{array}{ll}\text { le portiQue } & \text { Le Portique } \\ \text { Revue de philosophie et de sciences humaines }\end{array}$

$20 \mid 2007$

Gilles Deleuze et Félix Guattari : Territoires et devenirs

\title{
Visions croisées
}

\section{René Schérer}

\section{OpenEdition}

\section{Journals}

\section{Édition électronique}

URL : http://journals.openedition.org/leportique/1353

DOI : $10.4000 /$ leportique. 1353

ISSN : $1777-5280$

\section{Éditeur}

Association "Les Amis du Portique"

Édition imprimée

Date de publication : 15 décembre 2007

ISSN : 1283-8594

Référence électronique

René Schérer, « Visions croisées », Le Portique [En ligne], 20 | 2007, mis en ligne le 06 novembre 2009, consulté le 26 mars 2021. URL : http://journals.openedition.org/leportique/1353 ; DOI : https://doi.org/ 10.4000/leportique.1353

Ce document a été généré automatiquement le 26 mars 2021.

Tous droits réservés 


\title{
Visions croisées
}

\author{
René Schérer
}

1 Je suis heureux d'intervenir dans ce colloque consacré à Gilles Deleuze et à Félix Guattari ${ }^{1}$, deux amis très chers et très proches, dont le nom et les œuvres, depuis qu'ils nous ont quittés, ne cessent de m'accompagner et de m'insuffler. De me pousser à remonter en esprit à ce temps où leur pensée nous saisissait dans toute la force et la fraîcheur de son jaillissement.

2 Pour ceux qui n'ont pas vécu cette période, et je pense que c'est la plupart d'entre vous, essentiellement les étudiants, je rappellerai que j'ai été le collègue de Gilles Deleuze à l'Université de Vincennes, après qu'elle a été fondée en 1969, ainsi que de deux de ses amis qui ont présidé à la création du département de philosophie, chargés plus particulièrement de son aménagement: je veux dire Michel Foucault et François Châtelet. Le premier est resté peu de temps au département de philosophie de Vincennes, parce que, comme vous le savez, il est parti très rapidement au Collège de France ; l'autre était Châtelet qui est connu pour ses travaux sur La naissance de l'histoire, mais peut-être essentiellement, par sa grande Histoire de la Philosophie et celle des idéologies qui ont été mondialement diffusées, qui se sont assez vendues, et pour lesquelles il avait fait appel à la collaboration de beaucoup de philosophes, dont Gilles Deleuze. Dans l'histoire de la philosophie, Deleuze avait choisi, parmi les théories contemporaines, la rubrique concernant «le structuralisme»: théorie, une tendance plutôt, vers laquelle il avait penché plus ou moins pendant un certain temps avant de l'abandonner définitivement et de la critiquer, en particulier, à partir de ses relations avec Félix Guattari.

3 C'est-à-dire après 1969 ; l'article sur le structuralisme datant, si je me souviens bien, de cette année-là (mais rédigé, sans doute, plus tôt). Quant à l'ouvrage, les ouvrages battant en brèche l'influence et les idées mêmes du structuralisme, ce furent, on le sait, mais il convient ici de le rappeler, d'abord l'Anti-ÆEdipe et ensuite Mille plateaux, en collaboration avec Félix.

4 Or, comme ce colloque, d'après ce que j'ai saisi, est consacré un peu à égalité à Deleuze philosophe et à l'étude des relations et de la collaboration entre Gilles et Félix, je pense que là, avec l'évocation du structuralisme, nous sommes certainement au centre d'une 
notion qui répond d'une certaine manière à la question posée à travers l'exposé de François Fourquet et à propos de Guattari, sur la subjectivité et le sujet.

Peut-être avait-on laissé un peu dans l'ombre, justement, ce débat autour du structuralisme qui, à l'époque, prédominait. Un structuralisme qui, pour résumer d'un mot, était inspiré essentiellement de la linguistique saussurienne - il y avait sa source et avait, depuis, rayonné un peu dans tous les sens - qui visait, dis-je, à effacer en quelque sorte, à faire passer en second plan le sujet pour lui ôter son pouvoir au bénéfice du "système ». Un système formel constitué hors sujet, indépendamment de tout recours à une instance subjective; celle-ci, ou celles-ci (pour les sujets pluriels) prenant valeur et sens, au contraire, à partir du système.

Un des meilleurs exemples en est, certes, le système linguistique, avec ses marqueurs de subjectivité, relevant d'une structure préexistante. Mais ce n'était pas le seul. Le structuralisme s'était également implanté ailleurs. Il avait donné lieu à beaucoup d'élaborations dans diverses sciences, en particulier dans l'anthropologie : surtout avec Lévi-Strauss qui a écrit une Anthropologie structurale. Ou encore, dans la perspective et l'ambition d'une sémiotique générale, il y avait Roland Barthes, qu'aimait et admirait beaucoup, justement, Deleuze. Et il peut être pris, lui aussi, comme un exemple et un cas des plus remarquables de l'extension du structuralisme, en l'accommodant, d'ailleurs, de façon très libre et même critique ; en en retenant surtout le trait essentiel de l'abandon du sujet et de l'intériorité.

7 C'est ainsi que, ayant pour sa part déjà, dans ses écrits antérieurs, lancé de nombreuses attaques contre la philosophie spiritualiste, l'idéalisme de l'intériorité et du sujet, Deleuze a, dans l'article écrit pour Châtelet, été amené à présenter le structuralisme sous un jour favorable, à prendre parti en sa faveur contre les psychologies et les philosophies subjectives. Et à réserver, exemplairement, à l'interprétation structuraliste de la psychanalyse par Lacan, le meilleur des accueils, en raison de la façon dont la théorie lacanienne traite de la dissolution du moi et du sujet.

Cette critique structuraliste est comme un premier stade, un premier pas. Il est parfaitement exact que, dans l'Anti-৫́dipe, la théorie machinique se substitue à la théorie du sujet dans le domaine de l'inconscient; mais il faut ajouter qu'elle se substitue aussi à celle du structuralisme qui avait opéré, relativement au sujet, une première substitution.

9 Entre les théories du sujet, celles du structuralisme et celle d'un inconscient machinique qui vient les supplanter, il y a des relations complexes, puisque, dans une certaine mesure, le structuralisme est une théorie qui a présidé à l'effacement, à la mise à l'écart du sujet. Celui-ci y est réduit à une simple place, à une place vide, d'ailleurs; il n'est défini que par sa place, il est à localiser. Le sujet, ce n'est pas quelque chose qui domine ; ce n'est pas une instance dominante, une origine : c'est une instance qui, au contraire, est à situer, qui a à prendre place dans le système.

10 Il y a, dans le système linguistique, les opérateurs de subjectivité : par exemple, pour une langue, les pronoms, les pronoms personnels; et, dans le système psychanalytique qui est celui de l'inconscient, et qui d'ailleurs, à travers les interprétations qui en avaient été faîtes, à l'époque, par Jacques Lacan était mis en relation très étroite avec les systèmes linguistiques, ce sont les divers avatars du sujet relativement à un « ordre symbolique ", celui du signifiant, de la Loi, du Nom du Père, etc. 
11 Mais n'ayez crainte, pardonnez-moi, je ne vais pas me hasarder ici à tracer ou même à esquisser un historique de cet ensemble de problèmes complexes. Je voulais simplement rendre sensibles des interférences de doctrines et de systèmes. Tout en reliant ceci au titre-annonce de mon intervention qui mentionne des "Croisements". Et je crois que vous avez bien là une sorte d'illustration de cette notion de croisement qui occupe même différents étages, pour ainsi dire.

12 Autour de - et avec - Félix Guattari et Gilles Deleuze qui, au moment de leur rencontre, vont croiser leurs parcours et leurs points de vue, on voit simultanément le croisement d'un certain nombre de tendances, d'un certain nombre de systèmes majeurs, de conceptions dominantes dans la pensée contemporaine.

13 Croisement donc au niveau des théories de la structure linguistique, croisement au niveau des théories de l'inconscient, au centre desquels Felix et Gilles vont s'installer, avec leurs écritures entrecroisées.

14 Et qu'en résulte-t-il ? Une nouvelle manière de voir, de penser. En tirant du croisement une irruption dans autre chose. Pour parler bref, je dirai que, ces croisements, Félix Guattari et Gilles Deleuze, dans leur collaboration, les ont à la fois établis et dissous. Qu'ils ont tranché en eux, à partir d'eux, d'une façon péremptoire, d'une façon décisive. Qu'est-ce à dire?

Certes, ma formule est un peu boiteuse. L'image que j'utilise n'est pas très aisée à concrétiser.

Qu'est-ce que ça peut bien signifier que « trancher » dans un « croisement »?

18 Mais on voit pourtant à peu près ce que ça veut dire. C'est que dans des problématiques qui ne peuvent arriver à être décisives, soudain, brusquement, par un coup génial, on saute le pas, on passe ailleurs. On le voit très bien, déjà, en ce qui concerne Deleuze isolément, dans un ouvrage comme Logique du sens qui fut, par élection, il l'a déclaré quelque part, Psychanalyse et transversalité, je crois, la référence philosophique de Félix, ouvrage fondamental, génial, tout à fait remarquable, de ce point de vue de la rupture, $\mathrm{du}$ « trancher » dans le croisement, à l'instant où se produit un croisement.

19 Car, on y décèle comment, tout en reprenant un certain nombre de concepts et de termes de la psychanalyse dans les commentaires, par exemple, de l'oralité, c'est d'une tout autre manière que celle d'un psychanalyste que cela est traité.

20 Et on le voit, de même, dans l'étude sur Sacher-Masoch, avec la dissociation du complexe trivial du sado-masochisme, avec le passage au plan littéraire d'une critique clinique, au plan du contrat masochiste, une saute qui est comparable à une transmutation des valeurs, de type nietzschéen, une transvaluation.

21 Rupture et passage qui laissent, malgré tout, intact un certain vocabulaire que je dirais de transition, en tant qu'il reste un petit peu pris dans des concepts et dans des analyses de type encore freudien.

22 Et tout à coup, alors là, il y a une opération que je comparerai - en fait, je crois que la comparaison a une certaine pertinence -, avec l'expression, l'image que Nietzsche (encore lui) a utilisée à propos de ses propres œuvres - dans le Crépuscule des idoles - en parlant de : "philosopher à coups de marteau ».

23 C'est cela ce que j'appelle briser les croisements, ou trancher : c'est comme un coup de marteau asséné sur la structure ; et on introduit la notion de « machine ». C'est quelque chose qui, à ce moment-là, rompt définitivement avec les problématiques d'une 
philosophie du sujet, avec les impasses d'une pensée dans le cadre de la structure. On a rendu inutile la problématique, le faux problème, on a sauté le croisement.

Ce qui me conduit à une idée que je voulais exprimer ici, parce que je ne fais que parler à bâtons rompus et que je ne vais pas développer beaucoup de choses, mais indiquer certaines directions, peut être déblayer la voie pour certaines idées.

La première donc, je le répète, c'est celle du croisement dans la philosophie deleuzoguattarienne, entre un certain nombre de tendances qui sont les tendances de la philosophie contemporaine et où ils introduisent, tout à coup, brusquement, d'une façon violente si l'on peut dire, dans le bon sens de la violence, un concept qui va renouveler complètement la question et établir dans la philosophie contemporaine, ce qui, je crois, reste encore extrêmement valable : une notion vraiment révolutionnaire dans la conception de l'inconscient, avec cette idée d'un inconscient machinique. Même ceux qui n'adhèrent pas totalement à cette théorie, qui ne l'ont même pas pénétrée de façon complète, sont prêts à accepter justement des notions et expressions comme celle de machine, de machination, de fonctionnement machinique, lorsqu'il s'agit de choses qui dépassent incontestablement le domaine de la conscience, le domaine de l'intériorité, tout comme celui de la stabilité. La dynamique de la machine remplaçant la statique de la structure.

Le second point et qui est un point très important, je pense, dans la conception de Deleuze ainsi que dans celle de Guattari, c'est un peu la même chose mais exprimée dans d'autres termes, peut-être. C'est cette prise de position tout à fait particulière, singulière, qui caractérise leur philosophie propre dans un mouvement qui lui aussi déborde largement le cadre de cette philosophie. C'est justement celui de la crise, de la mise à l'écart, sinon de la destruction tout au moins de «l'exténuation » du sujet - je crois que ce mot n'est pas mauvais, il avait été employé par certains auteurs, vraisemblablement, selon mes souvenirs, Maurice Blanchot. Exténuation: le sujet est épuisé, à bout de course, il ne peut plus être utile pour la pensée.

C'est dire que la notion de sujet qui a dominé la pensée contemporaine depuis Descartes, avec Kant, en particulier et avec Hegel également -, puisque la dialectique de Hegel est placée sous le signe de la transformation de la substance de l'ancienne métaphysique en sujet -, c'est dire que cette notion en arrive à être tenue à l'écart, à ne se maintenir qu'en arrière-fond.

Sans doute, Deleuze et Guattari n'ont-ils pas été les seuls à avoir opéré cette mise en retrait, puisque déjà on en voit la contestation, l'effritement, avec les deux grandes théories qui tiennent le haut du pavé, pourrait-on dire, à cette époque. Deux, et même trois, d'ailleurs (quand on dit deux il y a toujours trois).

29 Ces deux grandes théories, vous l'avez compris mais je vais vous les dire tout de même, c'est la psychanalyse qui est une contestation du sujet fier et plein de lui-même, du sujet conscient ; et, d'autre part, en apparente contradiction avec elle, mais qui n'en a pas moins formulé une contestation du sujet : la phénoménologie - en général, en ce qui concerne la conception psychologique courante de la perception interne prétendant caractériser le sujet, et, en particulier, la phénoménologie de Heidegger pour ce qui est de la priorité de l'existence sur la conscience.

30 La troisième position puisque j'ai rappelé que «jamais deux sans trois », ce serait le marxisme, dominant, lui aussi en philosophie, à l'époque où Félix et Gilles sont intervenus. 
31 Il y avait donc des théories régnantes, des théories qui étaient dominatrices : la phénoménologie qui a été, vous le savez, lancée par Husserl, qui a été continuée et modifiée dans le sens de la phénoménologie existentielle, reprise en France par Sartre sous le nom d'existentialisme. L'une et l'autre considérées couramment comme des théories du sujet, bien qu'ayant entrepris leur critique; car il s'agissait en elles d'un sujet tout à fait particulier, tout à fait différent du sujet cartésien, du « Je pense donc je suis ", c'est-à-dire d'un sujet qui était profondément plongé dans l'existence, qui n'était pas du tout maître de lui-même totalement, qui était transformé en " être là ", qui était soumis à des situations, etc. donc c'était un problème, c'était une modification très profonde du sujet.

Et il y avait également la psychanalyse qui laissait le sujet conscient à l'écart en mettant au premier plan l'inconscient. Il y avait, enfin, le marxisme qui s'emparait du tout et qui à ce moment-là soumettait tout à un matérialisme dialectique.

Donc, un enchevêtrement de croisements, de diverses natures, parfois compatibles, parfois non. Quand je dis « visions croisées », les croisements dont je parle ne sont donc pas simplement entre Félix Guattari et Gilles Deleuze mais avec les théories qui sont en train de se croiser et là aussi il y a une manière qui a été tout à fait propre à leur mode de philosopher, à Félix et à Gilles, à leur manière de s'insérer dans ce croisement et de le trancher ou de taper dessus à coups de marteau en introduisant quelque chose d'autre.

34 Je me transporte maintenant un peu à côté, pour voir sous un autre angle et éclairer autrement la question, si possible. D'une façon qui m'est tout à fait propre et que je ne donne pas comme un modèle mais qui me vient à l'instant à l'esprit. Non un modèle ni une certitude, un simple exemple.

Je songe à une autre philosophie contemporaine qui est assez proche et qui, peut-être, tout en étant très différente dans ses origines et ses fins, me permettra, pour être bref, de vous donner un peu une idée, de l'orientation que j'aimerais donner, à présent, à ma réflexion.

Dans cet ensemble philosophique, donc, il m'arrive de songer à une théorie, à l'époque (les années 1970) un peu effacée, en retrait de ce mouvement révolutionnaire qui inspirait le deleuzo-guattarisme, mais qui a eu également son intérêt, son importance, et qui, à l'heure actuelle, occupe même peut-être, d'une certaine façon, une place prépondérante dans la pensée philosophique actuelle, celle d'Emmanuel Levinas.

J'en parle, parce que, sur la lancée et dans le développement de la phénoménologie, en particulier de la phénoménologie husserlienne, puis heideggérienne et de leur critique, il me semble qu'il a exprimé une idée et formulé des expressions tout à fait séduisantes, surprenantes, difficiles d'ailleurs à fixer d'une façon très précise, mais ouvrant, à coup sûr, des horizons et des voies extrêmement riches pour l'esprit : Autrement qu'être, audelà de l'essence. C'est le titre d'un de ses ouvrages, d'un recueil, justement, de rupture et d'inauguration.

C'est l'expression qui frappe, dans ses affinités certaines - à mon sens du moins - dans ses résonances du moins, avec l'entreprise qui lui fut contemporaine, de Guattari et de Deleuze.

Qu'est-ce que ça veut dire que cette chose-là : "Autrement qu'être, au-delà de l'essence »? 
C'est annoncer une philosophie, un type de philosophie qui va trancher avec le langage couramment utilisé. Qui affirme en avoir fini avec les notions traditionnelles, les appellations classiques des concepts. Surtout, au premier chef, avec les notions d'essence et "d'être » telles qu'elles se sont maintenues comme coextensives à «la Philosophie » (je mets des guillemets et une majuscule) de ses origines jusqu'à nous. Ce qui a permis, jusqu'au cœur des philosophies contemporaines, d'assurer la permanence et la haute dignité, la suprématie de «l'ontologie " (encore entre guillemets) sur toute autre forme de penser. Or, ce que dit la formule de Levinas, c'est qu'il faut dépasser cela, ce mode de penser qui ne convient plus.

«Au-delà de l'essence ». Ce n'est peut-être pas tout à fait neuf, puisque «l'essence » avait été déjà détrônée par Sartre au bénéfice de l'existence. Et il est certain que Levinas se trouve dans la lignée de l'existentialisme. Mais, dans la mesure où celui de Sartre, on s'en souvient, reposait sur la primauté du pour soi, ou, finalement, du sujet conscient, avec le passage à l'autre, avec l'affirmation de la priorité de l'autre, de cet irréductible dehors sur le dedans, de cette altérité primordiale, la formule de Levinas affirme la rupture. Comme, de leur côté, le font les auteurs de l'Anti-๕Edipe, elle donne le coup de marteau iconoclaste.

Elle opère à la manière de Nietzsche, en affirmant que l'existentialisme doit être surmonté.

3 Et, avec l'existentialisme, tout ce qui a rapport à l'être même. Car il ne suffit plus de se maintenir dans ces jeux d'opposition entre essence et existence ou être et existence, ou encore, comme l'avait fait Heidegger, de doubler l'existentiel'«ontique» d'un «ontologique» concernant l'authenticité d'un «être» supposé. Tout cet édifice spéculatif s'écroule.

4 L'idée survolante de "l'autrement qu'être " qui n'admet plus les notions classiques encore prépondérantes de l'activité du sujet et de la conscience de soi, et lui substitue celle d'une extériorité, d'une altérité, d'une passivité primordiales, introduit, dans l'image de la pensée, une rupture qui ne laisse pas intacte non plus le concept courant d'une ontologie.

Ici, et pour aller plus vite et plus loin, peut-être, du côté de quelque chose qui me semble très semblable à ce qui a lieu du côté de la pensée de Deleuze et de Guattari, je me limiterai simplement à cette invitation que fait Levinas de se porter au-delà, ou plutôt en dehors ou à côté de la pensée de l'être, d'abandonner la recherche, je dirais même la prétention ontologique.

Un déplacement, un coup de marteau sur l'être, le grand Être. Pan! Il se brise et tombe par terre. Levinas appelle cela l'anarchie. L'anarchie ou anarchisme philosophique. Bien sûr, il l'écrit an-archie, ce qui semble le démarquer de l'anarchie vulgaire, trop immédiatement subversive. Mais peu importe. Proudhon, d'ailleurs, écrivait aussi anarchie pour marquer l'absence de principe directeur centralisant, le défaut d'arché.

Pas d'arché, d'archonte, d'archétype, de hiérarchie dans les êtres. Tout se tient, tout se vaut. Simplement, « il y a ». L'expression est, elle aussi, levinasienne.

Et je vois là une grande parenté avec l'orientation deleuzienne. Sans doute Deleuze, dans Différence et répétition, semble-t-il maintenir la prérogative de l'ontologie en philosophie. Mais il ne faut pas s'en tenir au mot, mais aller voir de quoi il s'agit.

Daniel Colson, en exposant l'anarchisme de Deleuze, met l'accent sur quelque chose de très intéressant relativement aux attendus « ontologiques » de cet anarchisme, qu'il ne 
met pas, à la vérité, en relation avec celui exprimé par Levinas, mais avec «l'anarchiste couronné ", Héliogabale, d'Antonin Artaud. Quelque chose à quoi j'adhère d'une façon complète, tout en l'interprétant, pour ma part, comme une dissolution de l'ontologie.

C'est que, lorsque Gilles Deleuze affirme, à l'encontre les thèses scolastiques de la hiérarchie des êtres et de "l'analogie de l'être", entre celui de Dieu et celui des créatures, lorsqu'il affirme qu'il n'y a qu'une proposition ontologique et que cette proposition est celle de "l'univocité de l'être", il ne veut dire rien autre chose que ceci : l'être se dit de toute chose et se dit de toute chose de la même façon. Tout être est égal et équivaut à tout autre. Il est aussi bien celui d'un souffle, d'un rayon lumineux, que celui d'une pierre; " dieu, table ou cuvette », écrivait l'épicurien et gassendiste La Fontaine. Il n'y a que des êtres, pas d'être en soi, surtout pas.

51 C'est ça, je le pense, l'univocité de l'être dont parle Deleuze. Et c'est en ce sens qu'elle affirme, avec l'unité, non l'existence prétendue d'un être unique, mais la multiplicité, et l'anarchisme.

On peut invoquer, à ce propos, Épicure et Lucrèce, mais aussi Spinoza. Et, à cet égard, le commentaire de Deleuze, est très explicite, puisqu'il dit que la Substance une se résout tout entière dans les modes qui l'expriment. Pour Deleuze, il n'est pas question de concevoir quelque Être-un en soi indépendant des choses existantes.

Ce serait un peu de l'existentialisme, si vous voulez, par rapport à un essentialisme supposé.

Mais c'est aussi quelque chose de foncièrement différent ; c'est-à-dire que c'est quelque chose qui ne laisse même pas subsister, en un fond quelconque, cette opposition que l'on a sans cesse tendance à formuler de nouveau, entre essence et existence. Car, cette opposition essence/existence est elle-même prise dans un autre mouvement. Ou, pour mieux dire, elle est prise dans le mouvement, elle est prise dans le devenir.

Et, comme on a placé également ce colloque sous le signe du devenir, des devenirs, je crois qu'il y a là une source du devenir deleuzien, en rapport avec la "question ontologique », qui réside précisément dans cette affirmation que si l'un ne dit que du multiple, l'être ne se dit que du devenir.

Proposition qui est incontestablement aussi, si l'on y prête attention, celle de Nietzsche, et qui est, de plus, la façon dont cette philosophie va reprendre en compte ces différentes pensées à travers lesquelles elle pense et se pense. Ces auteurs auxquels il n'a pas estimé bon de "faire un enfant dans le dos ", mais auxquels, au contraire, il s'est totalement livré, qui l'ont totalement saisi. Deux ou trois auteurs que Deleuze reprend en compte et à travers lesquels il pense, qu'il « croise ", qu'il affirme aussi bien avoir exprimé sa propre pensée, que lui-même exprime aujourd'hui la leur. Oui, cette univocité, cette unité qui ne s'affirme que du multiple, et s'exprime dans le devenir, c'est bien l'idée de Nietzsche de la même façon que c'est l'idée de Spinoza.

Un croisement s'il en est, et qui, même, peut introduire une nouvelle remarque.

Car l'affirmation que l'un ne se dit que du multiple, que l'être ne se dit que du devenir, cette affirmation peut encore se comprendre et se placer sous le titre du croisement induit que l'être est, dans cette opération, en train de s'effacer. Sans trop forcer la note, on peut aussi comprendre le croisement en question comme une croix tracée sur l'être ; c'est-à-dire une rature de l'être, ou un être barré. Ce à quoi, il faut tout de même le rappeler, tendait aussi Heidegger et qu'il a proposé dans certains textes, se rendant 
compte du vide et du caractère finalement insoutenable et stérile de son "ontologie fondamentale ».

59

Faire une croix sur l'être. Sans l'avoir explicitement exprimé, je pense très sérieusement que c'est ce que propose Deleuze, avec son univocité de l'être et son passage au multiple.

Car, bien plus que la proposition d'une ontologie différente, il y a, chez lui, rupture totale avec l'idée d'une ontologie ou quelconque science de l'être. La prétention ontologique est vide. Et je pense encore que lorsque, dans une note du Pli, citant Gabriel Tarde, dans Sociologie et monadologie, Deleuze oppose à une "ontologie de l'être » une (inexistante et innommable, certes) « ontologie de l'avoir » ou de la relation, il formule sa véritable pensée sur ce sujet.

1 Et j'appelle cela une rupture totale avec l'ontologie, car il n'y a plus à ce moment-là du tout d'ontologie : c'est la déroute, la défaite de l'ontologie. La fin de l'ontologie qui était déjà préfigurée chez Heidegger à bout de course.

Jean Wahl, dont je vous recommande la lecture, avait écrit, vers 1968, je crois ou un peu après, seulement avec des références qui le faisaient encore prisonnier de la problématique classique, un livre très intéressant qui s'appelle Vers la fin de l'ontologie, qui mentionne précisément les problèmes et la croix, l'être barré dont je parle. L'intérêt de Deleuze pour qui le lit aujourd'hui dans cette perspective, c'est précisément aussi d'introduire là-dedans une rupture, non seulement de se contenter de l'entrecroisement de ces différents courants, mais de taper dessus à coup de marteau. La rencontre avec Guattari, leur collaboration furent également ici décisives : on brise cela et avec cela, on se tourne ailleurs, on parle d'autre chose que de l'être. Du désir. Surtout pas d'ontologie. Félix était en train d'opérer, de son côté, un même décrochage, de donner son coup de marteau dans le signifiant lacanien et sa structure, modalités ontologiques. Les deux façons vont tout à coup se conjoindre. C'est ensemble qu'ils vont désormais asséner les coups de marteau tombant à la fois sur l'être et sur le sujet, signant la déroute de l'ontologie. À remplacer par quelque chose d'autre. Leur « autrement qu'être ».

J'en suis donc amené à revenir à Levinas, dans l'intention, à présent, et pour terminer ce parcours, de marquer, avec la ressemblance, la différence. Ressemblance et différence aussi avec quelqu'un qui est un peu en marge de tous les mouvements de philosophie contemporaine, qui est Maurice Blanchot et qui a pensé aussi dans le même sens.

Seulement ils ont pensé d'une façon qui est assez différente et même très différente, si l'on songe à l'orientation fondamentale ou, pour mieux dire, plus exactement, car ce n'est pas tellement une question de vrai ou de faux, à la tonalité de leur philosophie. C'est cela : moins une question de vérité que de ton. De couleur ou d'accent.

Ici donc, c'est le dernier point que j'aborde. On va se trouver en face de quelque chose qui ne dépend pas d'une logique d'opposition classique qui va dire : « cela est vrai, cela est faux " mais d'une notion de tonalité. Deleuze a beaucoup insisté d'ailleurs dans Différence et répétition sur le fait qu'en définitive ce qui est important ce n'est pas tellement, ni essentiellement peut-être de savoir si quelque chose est vrai ou faux, mais de comprendre les valorisations qui sont au fond de tout cela. Et c'est assez nietzschéen. 

une instance supérieure ou en un arrière-monde; tout ce qui privilégie les valeurs de souffrance et de "passion ", de pitié, d'excessive humilité - ce que Fourier, pour sa part, a brocardé sous le chapeau : "l'amour du mépris de soi-même », un dénigrement de la vie des corps, tout ce pathos est éliminé d'une philosophie qui n'admet d'autre plan que celui d'immanence. Blanchot parmi les esprits prêtres, on ne peut s'empêcher de suspecter en eux, par l'entremise de l'expression de la transcendance, une certaine inclination vers le langage du mystère ou d'une indiscernable intériorité.

Or, je crois, avec François Châtelet qui en a parlé, que la première des transcendances c'est précisément la croyance à l'insondable d'un mystère intérieur, la supposition d'une intériorité, la construction d'une dimension de spiritualité incommensurable (le mot est de Levinas, il y est même fondateur) de ce qui est accessible au niveau de l'expérience sensible. La pensée de Deleuze est obstinément, il l'a exprimé lui-même, celle d'un empirisme transcendantal, où ce qualificatif ne signifie rien d'autre que 
l'élimination de toute transcendance. Comme chez Kant, au demeurant, ou, comme chez Husserl, la " réduction " du transcendant à l'immanence. Le paradoxal point commun qu'il garde avec la pensée critique de ces deux philosophes tout en restant, par bien d'autres aspects, à leur antipode.

L'incorporel n'est jamais susceptible d'être hypostasié sous la forme d'une réalité transcendante. L'invisible est l'envers d'un visible, non d'un autre ordre. D'un même et premier acte instaurateur, cette philosophie qui, encore une fois, comporte le passage intégral de l'être dans le devenir, la résolution totale de l'un dans le multiple, c'est également l'absorption radicale de tout le transcendant dans l'immanence. La composition du plan.

Et c'est là, dans ce qui va constituer une pragmatique d'un genre nouveau et pas du tout un avatar de l'ontologie, que Deleuze et Guattari se rencontrent d'une façon complète, sans qu'il y ait lieu de se demander quel est le philosophe entre les deux. Si c'est Deleuze que l'on dit être le philosophe, Félix Guattari est le psychanalyste : oui, il est toujours resté psychanalyste et Deleuze professeur de philosophie. Mais je crois que la philosophie de l'un et de l'autre se conjugue dans la construction, toujours à reprendre, de ce plan d'immanence qui est aussi « de consistance » ou d'élaboration des concepts. Leur commune condition de possibilité (ce que veut dire "transcendantal» dans « empirisme transcendantal »).

En quoi consiste la grande œuvre commune de Mille plateaux et qui est exprimée, d'ailleurs, à la fin de leur collaboration commune, tout au moins la dernière de leur collaboration commune, puisque la mort les a arrêtés en plein envol: Qu'est-ce que la philosophie?

Il n'y a rien d'autre que ce champ d'expérience, d'un type nouveau effectivement, par rapport aux empirismes classiques. Deleuze avait commencé par l'empirisme de Hume, et cette tendance empirique dans sa pensée n'a jamais cessé ; elle se nourrit, s'enracine, tire sa source, sa substance, sa matérialité de ce qu'il y a de pré-philosophique dans l'expérience : un chaos qu'il s'agit de composer, sans jamais abandonner le constat, le contact de la réalité empirique.

79 Il n'y a pas d'autre réalité que celle-ci, la philosophie est toujours restée pour lui une tâche, une tâche créatrice, elle n'est pas donnée, elle est en permanence à établir et justement par la violence faite à l'opinion commune, aux préjugés, aux transcendances qui n'en finissent jamais de renaître de leurs cendres. Transcendances revêtues de costumes variables mais bien reconnaissables sous ces travestissements qu'offrent la politique, la morale, les séductions d'un certain jargon de la profondeur; tout comme dans le domaine des institutions ou "segmentarités " c'est-à-dire les découpages habituels de la personne, de la famille, du sexe, etc.

Voilà à peu près en quoi je ferais consister ces différents croisements, ces croisements multiples qui ont constitué la philosophie de Gilles et de Félix, avec la nouvelle image de la pensée qu'ils ont élaborée en commun. Et que concrétisent, actualisent au mieux, pour le temps présent et notre futur aussi, les visions grandioses de Chaosmose, rejoignant les pages sur le « voyant» de Rimbaud écrites par Deleuze dans son Foucault. En cette fonction de voyance, les deux, les trois, se rencontrent, se croisent.

81 Je leur appliquerai volontiers aussi, pour conclure tout à fait, une expression, une répartition que je retiens, cette fois, de Foucault qui, dans un de ses séminaires de 1974 sur la naissance du Pouvoir psychiatrique, distingue, en une sorte de digression, deux 
formes, deux ordres de vérité: une vérité qui est une vérité supposée toujours présente, qui s'établit par voie de démonstration et une vérité qui ne jaillit, au contraire qu'à la faveur d'une rupture, d'un découpage brusque, en une vision rare et soudaine ; et qu'il appelle : « la vérité foudre », en l'opposant à la vérité démonstrative. Or, je crois qu'avec cette philosophie dite deleuzienne ou ces deux philosophies conjointes, de Deleuze et de Guattari qui se sont coalisées et croisées, coalisées pour bouleverser notre modernité, en produisant tant d'œuvres irremplaçables, nous avons affaire à ce genre de " vérité-foudre ", dont il est parlé.

\section{NOTES}

1. Il s'agit ici de la transcription quasi littérale de l'intervention orale prononcée dans le cadre du colloque par René Schérer à laquelle il n'a pas été cherché de donner un style écrit [Ndlr].

2. Il me faut ici compléter mon improvisation orale par la référence du livre: Autrement qu'être, au-delà de l'essence, Paris, Gallimard poche, 1990, chapitre IV, «La substitution. $1^{\circ}$ Principe et anarchie ", p. 157. Le texte est de 1967, et la première édition, chez Martinus Nijoff, de 1974. Je relève, entre autres : «Il ne s'agit pas pour le soi, dans son être, d'être. Au-delà de l'égoïsme et de l'altruisme, c'est la religiosité de soi » (p. 186).

\section{RÉSUMÉS}

René Schérer s'attache ici à mettre en évidence les divers croisements des travaux de Gilles Deleuze et Félix Guattari avec d'autres philosophies, pour montrer comment ils ont su «taper dessus à coup de marteau ", pour produire, en "tranchant ", une philosophie du désir qui rompt avec toute philosophie du sujet et toute ontologie.

Crossing sights René Schérer strives within this article to render visible the varied intersections of Deleuze and Guattari's efforts with other philosophies, and shows that they knew to "hit them with a hammer" to breach them, and to produce a philosophy of desire which breaks off from all philosophy of the subject and from all ontology.

Gekreuzte Ansichten René Scherer macht hier klar, wie sich die Arbeiten von G. Deleuze und F. Guattari mit anderen Philosophien überschneiden. Er will beweisen, wie damit eine Philosophie des Begehrens entstand, die mit jeder subjektiven und ontologischen Philosophie abbricht. 


\section{AUTEUR}

\section{RENÉ SCHÉRER}

René Schérer est Professeur émérite en philosophie à l'Université de Paris 8. Derniers ouvrages parus : Charles Fourier. Vers une enfance majeure, Paris, La Fabrique, 2006 ; Passages pasoliniens, en collaboration avec Giorgio Passerone, Lille, éd. du Septentrion, 2007-03-14 (à paraître en 2007) ; Après tout, entretien avec Geoffroy de Lagasnerie, Paris, Éd. Cartouche. En préparation : Méditations anarchisantes. Quelques mots pour Don Quichotte. 